\title{
Active Control of Cylinder Wakes: Use of Base Suction and Blowing
}

\author{
Y.Delaunay and L. Kaiktsis \\ Institute of Energy Technology \\ Department of Mechanical and Process Engineering \\ ETH Zentrum, Zurich, CH-8092, Switzerland \\ e-mail delaunay@lms.iet.mavt.ethz.ch
}

\begin{abstract}
The effects of active flow control, using base mass transpiration as control action, on the stability and dynamics of the cylinder wake at low Reynolds numbers are investigated, using numerical simulation. A spectral element method is used for the simulation. It is found that, in the subcritical Reynolds number regime $(\operatorname{Re}<47)$, slight suction destabilizes the wake, resulting in a vortex shedding pattern. In the supercritical regime, slight blowing stabilizes the flow, whereas high enough suction results in a steady and strongly asymmetric field. Further increase of the suction flow rate makes the flow steady and symmetric. The results are interpreted qualitatively, based on hydrodynamic stability considerations.
\end{abstract}

\section{Introduction}

Flows around bluff bodies have been extensively studied for more than a century, with the flow around a circular cylinder being the most well-known prototype. Detailed information (and references related to the problem) is given by Williamson [19], who has significantly contributed to this field.

In the absence of external forces, the dynamics of Newtonian incompressible flow around a cylinder depends only on the Reynolds number (here defined in terms of the free stream velocity and the cylinder diameter). Properties of this flow are well known for a wide range of flow regimes, from creeping flows $(R e \ll 1)$ to fully turbulent flows $\left(R e \approx 10^{7}\right)$. The flow regimes at low Reynolds numbers can be classified as follows:

For $R e<6$, the flow is steady and two-dimensional, with only one separation point, at the rear stagnation point.

In the range $6<R e<47$, the flow is still steady and two-dimensional, but a vortex pair appears behind the cylinder, whose recirculation length increases linearly with $R e$.

At $R e \approx 47$ a periodic vortex shedding is excited in the wake via a Hopf bifurcation [8]. In the absence of end-wall effects, the flow remains two-dimensional up to $R e=188.5$, and forms a dynamical system which can be described by the Stuart-Landau equation [14]. Note that the critical value of 47 is most accurately found by means of computational methods, which allow a perfectly two-dimensional flow.

For $R e>188.5$, the flow becomes three-dimensional via a secondary instability in the spanwise direction [1]. 
Flow control studies related to vortex shedding originated a few decades ago. It is well known that vortex shedding can cause structure damage through the oscillations of the flow, increase the drag and noise, but can also have advantageous effects, such as mixing and heat or mass transfer enhancement. All these issues are also relevant to more complex applications, than the simpler case of the circular cylinder considered here. Thus, since the experiments of Roshko [15], who by placing a splitter plate in the near wake of a cylinder suppressed vortex shedding, many experimental and computational studies have been performed in order to control wake flows at supercritical Reynolds numbers $[3,4,5,10,11,12,16,17,18]$. The most common methods (successfully tested at low Reynolds numbers) include splitter plates, wake heating, base bleed and suction, cylinder rotation or transverse oscillations, placement of a small cylinder in the near wake, and the use of steady magnetic fields.

In the present work, we investigate the effect of base suction and blowing on the stability of the cylinder wake at low Reynolds numbers, using numerical simulation. The results are interpreted based on hydrodynamic stability theory. The developments in hydrodynamic stability theory, with the introduction of the concepts of absolute and convective instability (e.g. see Huerre and Monkewitz[7]), has made vortex shedding more comprehensive, because of its close dependence on the stability properties of the base flow. The flow behind a bluff body contains two forms of local instability: the near wake velocity profiles are typically absolutely unstable, while the far wake profiles are convectively unstable. Thus, one can think of the vortex shedding process as the saturated non-linear state of a global instability triggered by the absolutely unstable region. As in other types of flows, the existence of a region of local absolute instability in the wake is not a sufficient condition to turn the base flow globally unstable. Chomaz et al. [2] propose a qualitative criterion for the existence of oscillatory patterns in a spatially developing flow, depending only on the absolutely unstable region :

$$
\int_{X_{a}}^{X_{b}} \sqrt{\sigma_{0_{i}}(x)} d x \geq O(1)
$$

where $\sigma_{0_{i}}(x)$ denotes the local linear temporal growth rate corresponding to zero group velocity, and $\left[X_{a}, X_{b}\right]$ the streamwise extent of absolute instability. This formula shows that a "global intensity" of absolute instability can be defined by integrating the square root of the local growth rate over the length of the absolutely unstable region; this quantity must exceed a certain threshold to excite vortex shedding in wake flows.

In the case of wake flows controlled by suction or blowing, one can interpret the flow's global stability state by considering the modification of the near wake profiles, and its implications on the flow's stability properties. The suction applied (e.g. at the cylinder base) tends to decrease the length of the recirculation zones, and thus decreases the streamwise extent of absolute instability. On the other hand, suction also increases the magnitude of negative velocities, which acts in favour of instability increase in terms of local temporal growth rate increase. Thus, the effect of suction on the wake is twofold: it increases the local linear growth rates in the near wake, and decreases the streamwise extent of absolute instability. Note that the effects of blowing are opposite to those of suction: linear temporal growth rates in the near wake are expected to decrease, due to decreased back flow, and the extent of absolute instability is expected to increase. Therefore, in both cases, one has to rely on phenomenological observations, to determine which action prevails for a given combination of the problem parameters. These competing effects due to suction or blowing require detailed investigation and have motivated the control studies of cylinder wake presented here. 
The present work uses numerical simulation to study the control of the cylinder wake in the steady and in the 2 -D oscillatory regime (here: $0<R e \leq 90$ ), by means of base suction or blowing. We report detailed results of flow states obtained within a broad range of Reynolds numbers and non-dimensional suction/blowing flow rates. In section 2, we define the problem set-up, and refer to the numerical methods used. In section 3 , we present our simulation results, and conclude in section 4 .

\section{Problem Definition}

\subsection{Governing equations, numerical method and discretization}

We consider a cartesian coordinate system $(x, y)$, with velocity $\mathbf{v}=(U, V)$, and solve numerically the 2-D time-dependent Navier-Stokes equations for Newtonian incompressible viscous flow, written here in non-dimensional form.

Momentum equation :

$$
\frac{D \mathbf{v}}{D t}=-\nabla p+\frac{1}{R e} \nabla^{2} \mathbf{v}
$$

Equation of continuity :

$$
\nabla \cdot \mathbf{v}=0
$$

where $\mathbf{v}$ is the velocity vector, $p$ the static pressure, and here $D$ denotes total derivative. Physical variables (length, time, velocity, and pressure differences) are non-dimensionalized with proper scales, based on the cylinder diameter $D$, the free stream velocity $U_{\infty}$, and the fluid density $\rho$. The Reynolds number is defined as

$$
R e=\frac{U_{\infty} D}{\nu}
$$

where $\nu$ is the kinematic viscosity of the fluid.

The spatial discretization is based on a Legendre spectral element method [13], while the time integration is based on a second-order accurate mixed stiffly stable scheme, with proper high-order boundary conditions for the pressure [9].

The computational domain and its decomposition into spectral elements is shown in Fig. 1. Here the origin is taken at the cylinder center. The following conditions are prescribed at the domain boundaries:

inflow and lateral boundaries :

$$
U=1, V=0
$$

outflow boundary :

$$
p=0, \frac{\partial U}{\partial x}=\frac{\partial V}{\partial x}=0
$$

(velocity boundary conditions at outflow being only imposed in weak form).

ESAIM: Proc., Vol. 7, 1999, $106-119$ 
The computational domain extends $16 D$ upstream from the cylinder center and $25 D$ downstream. The lateral boundaries both lie $12 D$ away from $y=0$; we assume that, at this distance from the cylinder, the velocity is only slightly affected by the presence of the bluff body, and can therefore be approximated by the free stream velocity. The domain is divided into 260 spectral elements, with $7 \times 7,9 \times 9$ or $11 \times 11$ collocation points per element. A particularly fine mesh is prescribed near the region of control action (see Fig. 1), to resolve the high velocity and pressure gradients present. $P$-refinement tests have been performed for several representative cases, to verify the independence of results on spatial resolution.

At present, we perform detailed investigations on the effect of the computational domain size on the computed fields. Several domains are used, the largest extending $30 D$ upstream and $36 D$ downstream from the cylinder center, with the distance of each of the lateral boundaries from $y=0$ being $34 D$. Results indicate that the qualitative picture given by the transitions reported in the present paper is not affected, while the critical values may change by approximately $10 \%$.

Depending on the physical parameters and spatial resolution, a time step ranging from 0.001 to 0.01 is necessary, imposed in most cases by numerical stability (and not accuracy) constraints. The time-histories of the velocity components and pressure are followed at five representative "observation" points (see Fig. 1).

\subsection{Control action definition}

Suction or blowing is applied on a small section of the cylinder surface, close to the rear stagnation point, corresponding to $\alpha_{0}=22.5^{\circ}$ (see Fig. 2). Physically, this configuration is an approximation of the experimental set-up used in Schumm et al. [17], where a wider region with $\alpha_{0}=30^{\circ}$ was used. Here, the suction/blowing velocity is specified on the cylinder boundary, i.e. on the circle, and the direction of the velocity vectors is radial. Thus, the present set-up can also correspond to a cylinder with a porous part in the rear stagnation point region. The radial suction/blowing velocity profile prescribed is uniform over most of the arc used; a smooth (cubic function of the angle, with zero derivatives at the two boundaries) profile is prescribed close to $\alpha= \pm \alpha_{0}$, bringing the velocity to zero, see Fig. 2.

Based on the prescribed suction volume flow rate $Q_{\text {suc }}$ and a reference flow rate, $Q_{\text {ref }}=U_{\infty} D$, we define a suction coefficient as follows :

$$
C_{\text {suc }}=\frac{Q_{\text {suc }}}{Q_{\text {ref }}}
$$

The dynamics of the flow under control action depends on two critical parameters: the Reynolds number $R e$ and the suction coefficient $C_{s u c}$. Note that, here, positive suction coefficient values correspond to suction, and negative values to blowing.

\section{$3 \quad$ Numerical simulations}

As previously mentioned, we expect that the wake stability is affected by the use of suction and blowing, both at supercritical and subcritical Reynolds numbers. Our first objective is to calculate asymptotic states for a large number of parameter sets $\left(R e, C_{\text {suc }}\right)$. To this end, we 
proceed as follows: uncontrolled flows $\left(C_{\text {suc }}=0\right)$ are first calculated for several Reynolds numbers. Subsequently, the control action is progressively increased, i.e. $C_{s u c}$ increased or decreased, by a step of 0.2 for suction or -0.05 for blowing. When a transition occurs, the precise value of critical suction coefficient is approximated by a dichotomy approach. The maximum absolute error in this value is then about 0.01 . The computed flow states in the parameter plane are summarized in Fig. 3. For a given Re, circles correspond to computed steady flows, closest to the critical curves. The flow states corresponding to no control action are indicated by the dashed horizontal line, which crosses the critical curve at $R e=47$.

As illustrated in Fig. 3, in the subcritical Reynolds number regime, we find that suction leads first to unsteadiness, and then again to a steady flow at high enough suction coefficient values (over 1.6 or so). In this regime, blowing has no effect on the wake stability. For $R e<17$, no control action can render the wake unsteady. At supercritical Reynolds numbers, even small blowing flow rates stabilize the wake, whereas much stronger suction is necessary to achieve this goal. The critical suction coefficient values corresponding to wake stabilization by blowing are in good agreement to the ones found by Schumm et al [17]. They report an asymptotic value of 0.12 for their blowing coefficient (based on the same reference flow rate as in equation 7), below which the wake is steady for all $R e$ below 175. However, they find a higher threshold value $(R e=27)$, below which the wake cannot become unsteady. This limit in Re given by Schumm et al. is based on observations with rather low suction coefficient values (around 0.6-0.7). The highest suction coefficient they tested is about 0.62 at $R e \approx 30$, which is very close to the critical value for $C_{\text {suc }}$ we also find at $R e=30$. Since this discrepancy between experiments and the present computations can be the result of difference in the suction boundary conditions, we also performed simulations with a suction velocity profile defined on a straight boundary, corresponding to an angle $\alpha_{0}=30^{\circ}$ (see Fig. 2), instead of the arc which follows the cylinder shape. With this new set-up, we could also destabilize the wake for Reynolds numbers below 20 .

The high critical suction coefficient values in the case of suction suggest that blowing is a much more efficient method for stabilizing the wake. For the Re range considered here, the minimal critical suction coefficient rendering the wake steady is 0.86 , and is obtained at the highest $R e$ value studied, $R e=90$. This is a consequence of the fact that the critical suction coefficient decreases with Reynolds number (see Fig. 3); this is surprising, as one expects the control effort to increase with Reynolds number. We believe that the observed trend is due to the increased effect of suction on the non-parallel nature of the base flow with increasing $R e$; note that non-parallel effects can significantly reduce the global growth rate in wake flows, see Leu and Ho [10]. We expect that the curve will tend to an asymptote for Reynolds numbers increasing beyond 90 .

Figure 4 illustrates the effect of suction and blowing on the wake, in terms of the non-dimensional frequency (Strouhal number) of the vortex shedding limit cycle. For all Re, the Strouhal number decreases with increased suction. The decrease of Strouhal number with suction is steeper at higher $R e$, and thus the function $S t(R e)$ becomes decreasing at high suction coefficients, whereas it is increasing for the uncontrolled flow. These values confirm the dramatic effect of suction on the flow structure, and also indicate that the flow is more sensitive to suction at higher Reynolds numbers; this results in the previously mentioned earlier stabilization of the wake at higher Reynolds numbers.

To understand the flow pattern, in addition to St, the amplitude of velocity fluctuation is also an important parameter. A global intensity of flow oscillation can be expressed in terms of the maximum value of the RMS fluctuation intensities $\left(u_{\max }^{\prime}\right.$ and $\left.v_{\text {max }}^{\prime}\right)$ over the whole domain. These values are presented in Fig. 5 as function of the suction coefficient, for $R e=90$. Clearly, there is 
an increase of fluctuation intensities with $C_{\text {suc }}$, until they reach a maximum close to the critical value of the suction coefficient.

Based on Figs. 4 and 5, we expect that, at suction coefficient values close to the critical, the flow structure is very different from that in the uncontrolled case. The time-history of V-velocity at point A (located at $x=1.5, y=0.0$ ), for $R e=90$ and $C_{\text {suc }}=0.85$ (slightly below the critical value), see Fig. 6, shows that suction tends to make the flow intermittent and amplify fluctuations. A comparison with the velocity signal of the uncontrolled flow, also presented in the same Figure, shows that, close to the critical suction coefficient value, the shedding period is about ten times larger, and the V-velocity amplitude twice as large as those of the uncontrolled flow. The strong modification of vortex shedding with large suction is illustrated in Fig. 7, where we present a sequence of instantaneous vorticity isocontours over one shedding period, for $R e=80$ and $C_{\text {suc }}=0.8$. The large increase in shedding period results in the formation of distinct vortices in the flow domain. Here the shape of each vortex is not affected by the presence of other vortices, as in the uncontrolled flow; thus the vortices acquire a circular form.

In contrast to suction, blowing at supercritical Reynolds numbers has a stabilizing effect on the wake, and damps oscillations rather smoothly (see Figs. 5 and 6). For the same flow rate, blowing has a stronger effect in modifying the Strouhal number of vortex shedding (see Fig. 4). It is interesting to note that, at subcritical $R e$, the effect of suction on the flow dynamics is different, even for high suction coefficients. In Fig. 8, we present the $\mathrm{V}$-velocity time-history at point $\mathrm{A}$, for $R e=30$ and $C_{\text {suc }}=1.58$ (which is 0.01 below the critical value). By comparing Figs. 6 and 8 , we find that the transversal velocity fluctuation amplitude is not as large as for higher Reynolds numbers; in addition, fluctuations are very regular and not intermittent in nature.

The transition from unsteady to steady flow at high values of $C_{s u c}$ is characterized by a simultaneous transition to asymmetric flow. The time variation of the $\mathrm{V}$-velocity component at point $\mathrm{A}$ (located on the domain centerline), as $C_{\text {suc }}$ is impulsively increased from 0.80 to the supercritical value of 1.0 at $R e=90$, is presented in Fig. 9. The sudden increase of $C_{\text {suc }}$ (indicated by the dashed line) results in suppression of flow unsteadiness, with the $\mathrm{V}$-velocity at the centerline converging to a steady non-zero value, representative of flow asymmetry. Our results indicate that, in this region, the symmetric solution is unstable; two stable mirror-image solutions are found, corresponding to strongly asymmetric flow. Convergence to either one of the two solutions depends, of course, on the simulation initial conditions; usually those are defined from an instantaneous (unsteady) field, at a lower $C_{\text {suc }}$ value. Fig. 10 shows the streamline pattern corresponding to one of the two asymmetric solutions for the parameter set $R e=90, C_{s u c}=1.0$, indicating a strong deflection of the front stagnation point and overall flow asymmetry. No separation from the cylinder surface is present. The presence of an asymmetric flow structure in a perfectly symmetric set-up is evidently associated with a non-zero value of the lift coefficient. Quantitative data of the drag and lift coefficients are presented below.

Transition from unsteady to steady and asymmetric flow is only observed for $R e>41$. Below this value, the steady flow obtained (by further increasing $C_{\text {suc }}$ ) is symmetric (see Fig. 3). For given $R e$, the steady asymmetric flow undergoes a transition to a steady symmetric state, at a second critical value of the suction coefficient. Thus, in the $\left(R e, C_{\text {suc }}\right)$ plane, there is a second critical curve, separating asymmetric and symmetric steady flow states (see Fig. 3). Careful processing of computed flow data has, in some cases, indicated signs of slight flow asymmetry (e.g. lift coefficient values values of the order of $10^{-3}$, clearly above machine accuracy), for flow states above the second critical curve. This issue is currently under investigation. Interestingly, in the Re range $(30,40)$ the flow undergoes four transitions for $C_{s u c}$ increasing from 0 to about 2.2 . 
Fig. 11 shows a bifurcation diagram for $R e=90$. Here, we plot the time-averaged value of Vvelocity at the centerline point $\mathrm{A}$, as a function of the bifurcation parameter, $C_{\text {suc }}$. For steady flow, the time-averaged value coincides with the asymptotic value. The flow is unsteady and symmetric in the mean for subcritical values of the suction coefficient, so the time-averaged V-velocity along the centerline is zero. After the first bifurcation $\left(C_{\text {suc }} \geq 0.86\right)$ two stable, steady and asymmetric (mirror-image) solutions exist, and the centerline $\mathrm{V}$-velocity can be positive or negative. At a second critical value, $C_{\text {suc }}=1.65$, the flow bifurcates to a steady and symmetric state. We find that the second bifurcation is characterized by a hysteresis effect; thus, within a narrow range of $C_{\text {suc }}$, both the symmetric and asymmetric solutions are stable and can be realized physically. The hysteresis regime is also indicated in the map of flow states (Fig. 3). Note that, since the asymptotic flow states, corresponding to the steady symmetric and asymmetric solutions, differ significantly in structure, using either one of them as initial condition to converge to the other one, results in very long transients, which exceed the typical shedding period of the uncontrolled flow (of about 6 units) by more than three orders of magnitude.

The flow asymmetry can be expressed in terms of the deflection of the front stagnation point, with respect to the centerline. The precise location of the stagnation point can be determined from the pressure maximum on the cylinder surface. In Fig. 12, we plot the angle (in degrees) formed between the centerline and the line connecting the stagnation point and the cylinder center, as function of $C_{s u c}$, for $R e=90$. The two transitions are indicated by dashed lines. This Figure clearly illustrates the abrupt transition to asymmetric flow, and also shows that asymmetry is more pronounced in the range of $C_{\text {suc }}(1.2,1.5)$.

The forces exerted by the fluid on the body are expressed in non-dimensional form by the drag and lift coefficients. We calculate drag and lift by integrating over the full cylinder surface, i.e. we consider the case of a porous cylinder. Computed values at $R e=90$ (pressure and viscous components, and total values) are presented in Figs. 13 and 14, as functions of $C_{\text {suc. }}$. Here, we report time-averaged values of the drag coefficient; since the time-averaged lift coefficient is zero in unsteady flow around a cylinder, the reported lift coefficient for (saturated) unsteady flow corresponds to its amplitude, i.e. half of the difference between its maximum and minimum values. We have checked our results corresponding to uncontrolled flow $\left(C_{s u c}=0\right)$, by comparing them to validated literature data (e.g. Henderson [6]), and found deviations of less than $2 \%$.

As shown in Fig. 13, the drag coefficient is minimal at the critical blowing flow rate $\left(C_{\text {suc }}=-0.14\right)$, and increases monotonically with suction coefficient in the unsteady flow regime. The decrease (by $7 \%$ ) in total drag at the transition from unsteady to steady asymmetric flow is due to the decrease in pressure drag. The rapid increase in pressure drag with $C_{s u c}$ in the steady (asymmetric and symmetric) flow regime results in a corresponding increase in total drag (see Fig. 13).

The lift coefficient is also monotonically increasing with $C_{\text {suc }}$, both in the unsteady and in the steady asymmetric flow regimes (Fig. 14). In all cases, the pressure component is most significant. In the unsteady flow regime, due to the phase shift between the instantaneous pressure and viscous lift, the sum of the two component amplitudes does not equal the amplitude of total lift (see Fig. 14). A discontinuous decrease in total lift (caused by the decrease of both viscous and pressure lift) is observed at the transition from unsteady to steady asymmetric flow. We emphasize the pronounced effect of suction on the lift coefficient: the values corresponding to asymmetric steady flow may exceed the time-averaged drag coefficient of the uncontrolled flow by an order of magnitude. 


\section{Summary}

In the present work, we have investigated the effects of base suction and blowing on the stability and dynamics of the flow around a circular cylinder, at low Reynolds numbers, using numerical simulation. Our results show that, at supercritical Reynolds numbers, slight blowing can stabilize the wake, as result of reduced back flow, and, thus, reduced absolute instability in the near wake. High enough suction can also stabilize the wake at supercritical Reynolds numbers, due to decrease in the streamwise extent of absolute instability. Flow stabilization by means of base suction results in a strongly asymmetric flow pattern. The steady asymmetric flow bifurcates to a steady symmetric state at higher suction flow rates.

At subcritical Reynolds numbers, sufficient suction can result in global instability and flow unsteadiness, due to increased back flow, and, thus, increased absolute instability in the near wake. With the present definition of control action, no control measure can render the wake unsteady for Reynolds number values below $R e=17$.

Since low levels of blowing are enough to stabilize the wake at supercritical Reynolds numbers, overall, blowing does not affect the shedding frequency significantly. On the other hand, suction can drastically reduce the Strouhal number and can strongly modify the vortex shedding process. Suction also increases the drag coefficient, while blowing results in decreased drag coefficient levels, until a steady flow is obtained. Thus, taking into account the difference in the required flow rates for wake stabilization, blowing is energetically a much more efficient method for stabilizing the wake, than suction. Suction is only preferable when a constant non-zero value of the lift coefficient is desired.

In work still underway, we investigate the effects of domain size and boundary conditions on the transitions reported in this paper. First results indicate that the same flow regimes are present, while their boundaries are affected by the choice of domain size and boundary conditions. Detailed data will be reported later.

Acknowledgments: Financial support for the present work was provided by ETH-Zürich. The computations were performed on the CRAY J-90 and on the Convex Exemplar SPP2000/X-32 of ETH-Zürich. We would like to thank Professors P. Koumoutsakos, L. Guzzella and A. Tomboulides for helpful discussions.

\section{References}

1 Barkley, D. And Henderson, R.D.: Three-dimensional Floquet stability analysis of the wake of a circular cylinder. J. Fluid Mech., 322 (1996), 215.

2 Chomaz, S.C.R., Huerre, P. And Redekopp, L.G.: The effect of nonlinearity and forcing on global modes. In New Trends in Nonlinear Dynamics and Pattern-Forming Phenomena (ed. P. Coullet and P. Huerre) (1990), 259

3 Hammond, D.A. And Redekopp, L.G.: Global dynamics of symmetric and asymmetric wakes. J. Fluid Mech., 331 (1997), 231.

4 Hammond, D.A. And Redekopp, L.G.: Global dynamics and aerodynamic flow vectoring of wakes. J. Fluid Mech., 338 (1997), 231.

5 Hannemann, K. And Oertel, H.: Numerical simulation of the absolutely and convectively unstable wake. J. Fluid Mech., 199 (1989), 55.

6 Henderson, R.D.: Details of the drag curve near the onset of vortex shedding. Phys. Fluids, 7(9) (1995), 2102. 
7 Huerre, P. And Monkewitz, P.A.: Absolute and convective instabilities in free shear layers. J. Fluid Mech., 159 (1985), 151.

8 JACKSON, C.P.: A finite-element study of the onset of vortex shedding in flow past variously shaped bodies. J. Fluid Mech., 182 (1987), 23.

9 Karniadakis, G.E., Israeli, M. and Orszag, S.A.: High-order splitting methods for the incompressible Navier-Stokes equations. J. Comp. Phys., 97 (1991), 414.

10 LEU, T.S. AND Ho, C.M.: Control of global instability in a non-parallel near wake. Submitted J. Fluid Mech., (1996).

11 Mutschke, G., Gerbeth, G., Shatrov, V. and Tomboulides, A.: Two- and three-dimensional instabilities of the cylinder wake in an aligned magnetic field. Phys. Fluids, 9(11) (1997), 3114.

12 Nishioka, M. AND SATo, H.: Mechanism of determination of the shedding frequency of vortices behind a cylinder at low Reynolds numbers. J. Fluid Mech., 89 (1978), 49.

13 PATERA, A.T.: A spectral element method for fluid dynamics; laminar flow in a channel expansion. J. Comp. Phys., 54 (1984), 468.

14 Provansal, M., Mathis, C. and Boyer, L.: Benard-von Karman instability: transient and forced regimes. J. Fluid Mech., 182 (1987), 1.

15 Roshko, A.: On the wake and drag of bluff bodies. J. Aero. Sc., 22 (1955), 124.

16 Roussopoulos, K.: Feedback control of vortex shedding at low Reynolds numbers. J. Fluid Mech., 248 (1993), 267.

17 Schumm, M., Berger, E. And Monkewitz, P.A.: Self- excited oscillations in the wake of twodimensional bluff bodies and their control. J. Fluid Mech., 271 (1994), 17.

18 Strykowski, P.J. And Sreenivasan, K.R.: On the formation and suppression of vortex 'shedding' at low Reynolds numbers. J. Fluid Mech., 218 (1989), 71.

19 Williamson, C.H.K.: Vortex dynamics in the cylinder wake. Ann. Rev. Fluid Mech., 28 (1996), 447. 

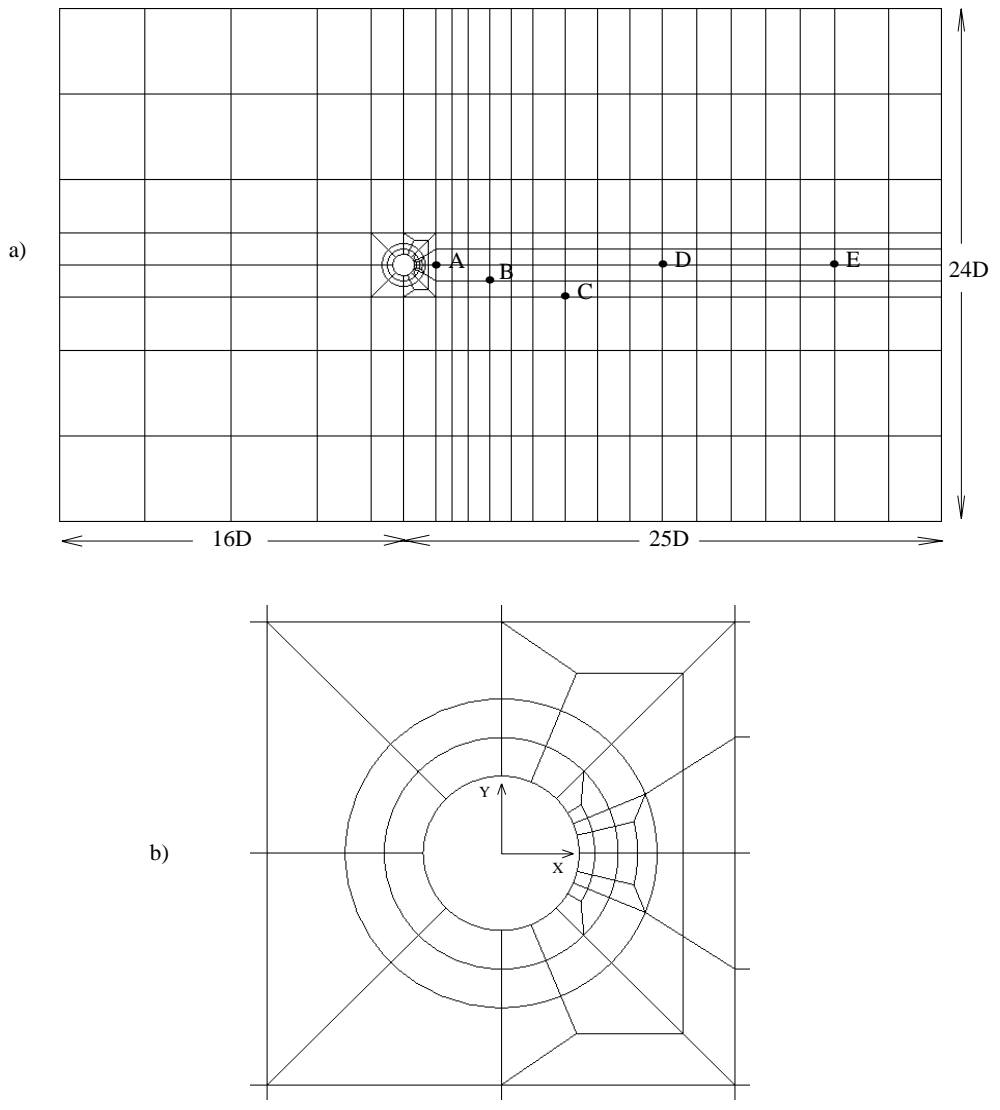

Figure 1: Spectral element skeleton for flow around a circular cylinder: a) entire mesh, b) elements close to the cylinder. Also shown are the five diagnostic points, where physical quantities are recorded.

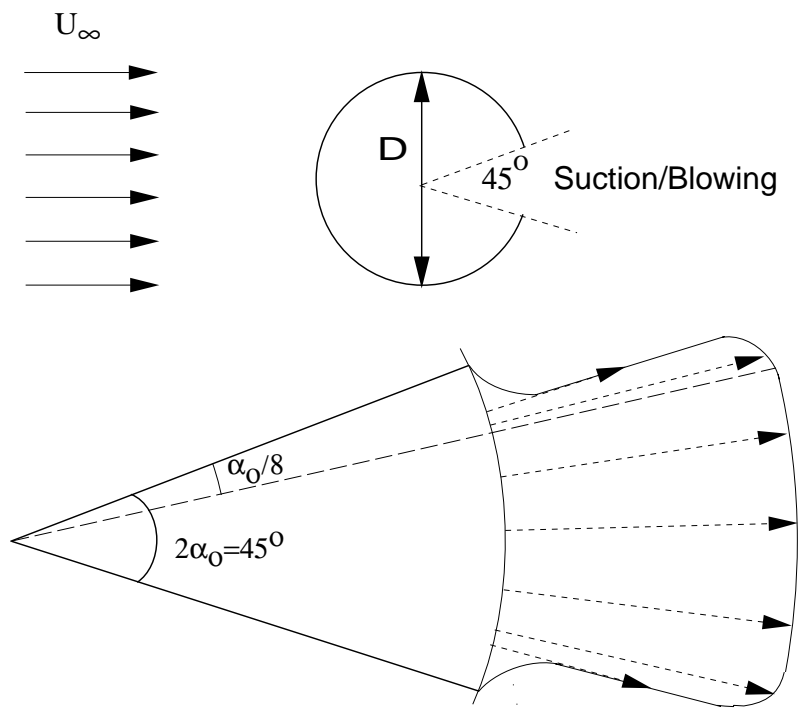

Figure 2: Sketch of the flow set-up with control action (here only the blowing case is illustrated). 


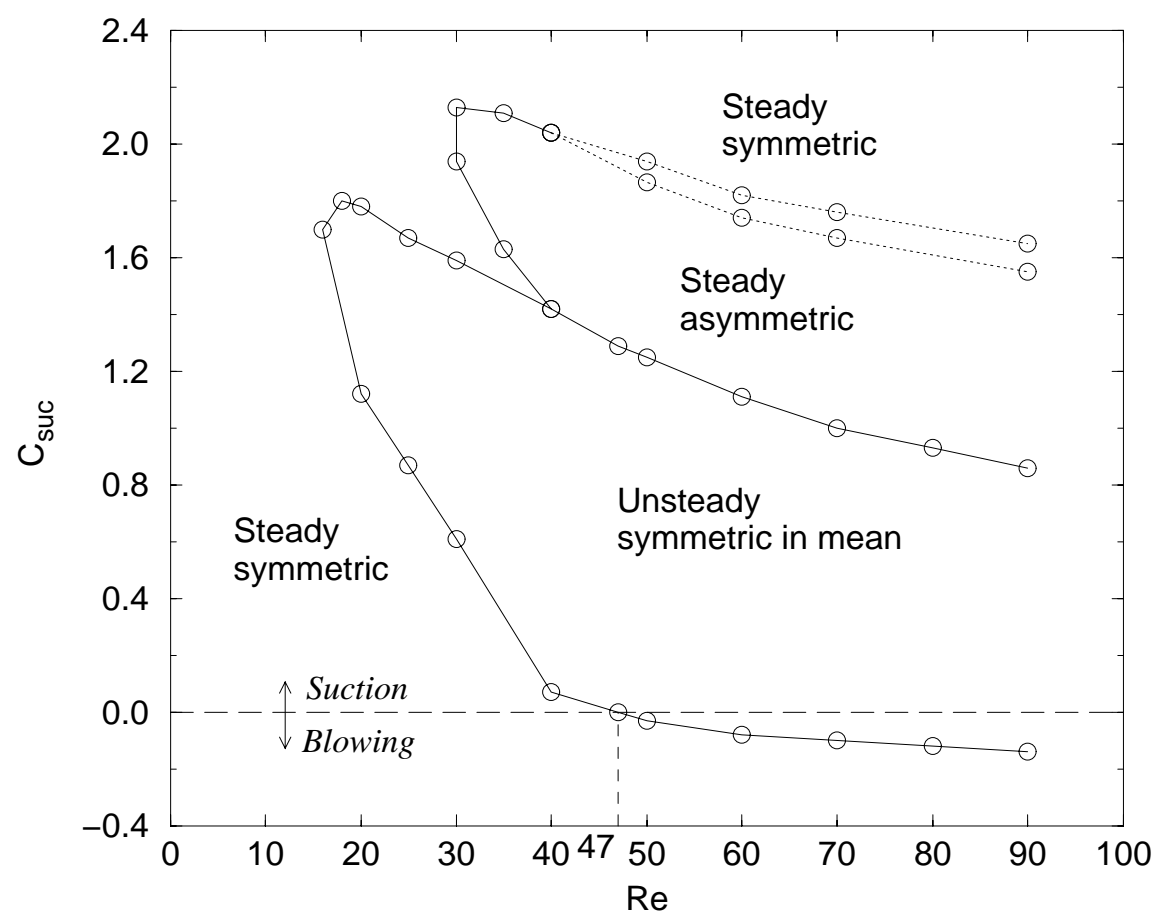

Figure 3: Flow states and corresponding critical curves in the parameter plane, $R e-C_{\text {suc }}$.

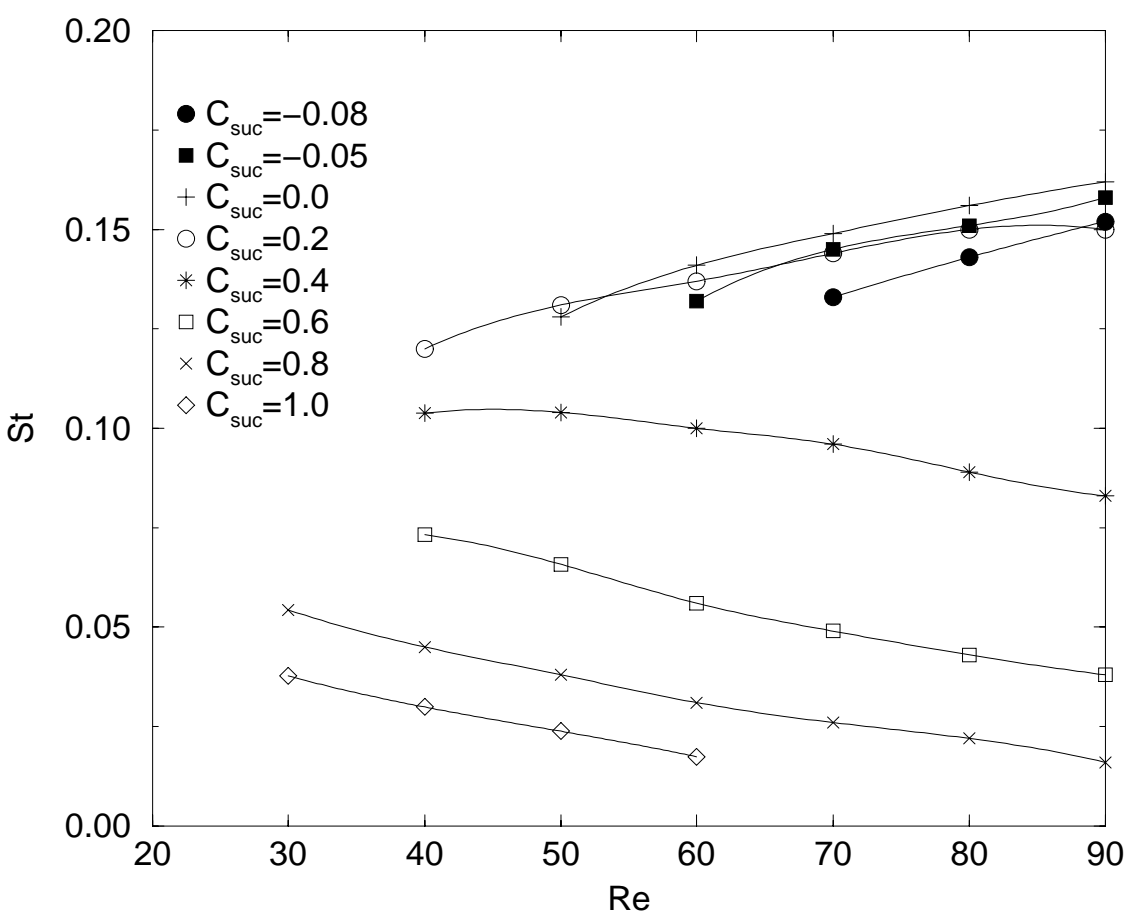

Figure 4: Strouhal number versus Reynolds number for different suction coefficient values. 


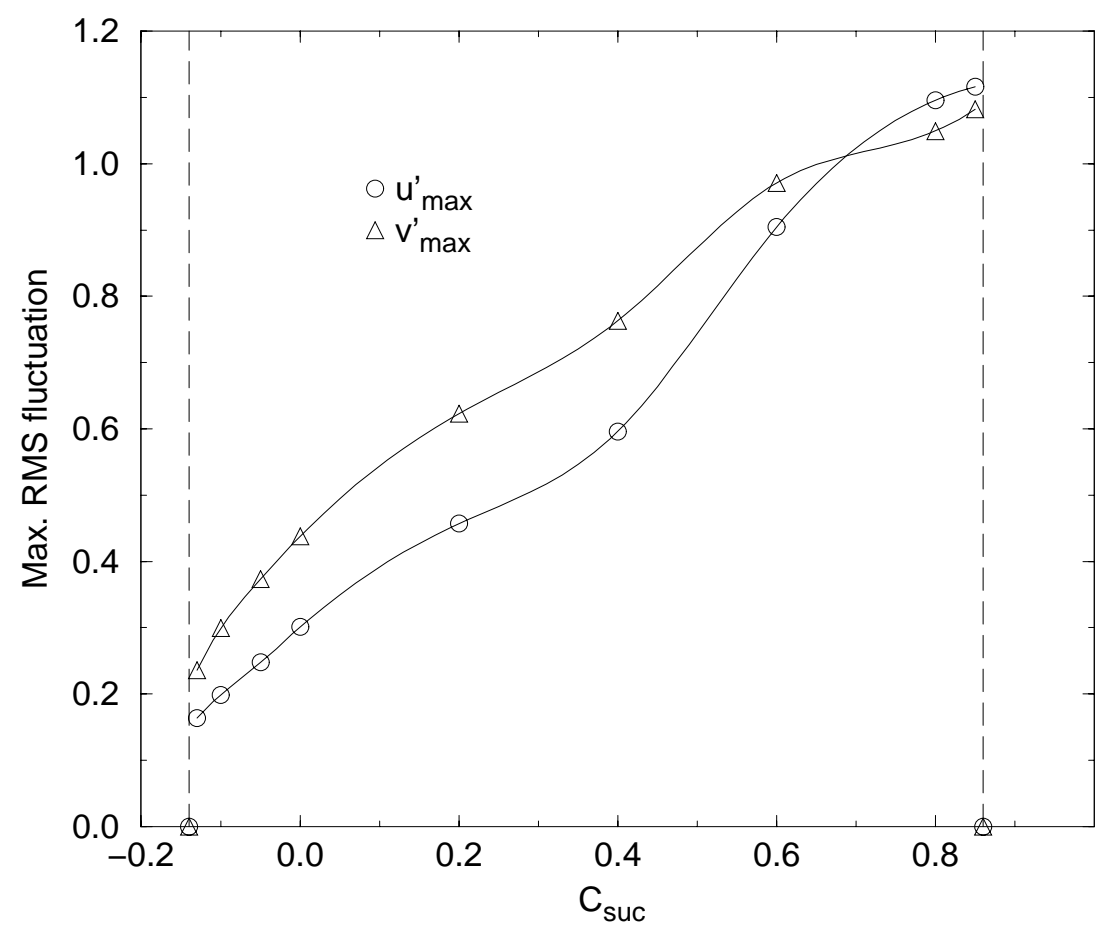

Figure 5: Maximal values of the fluctuation intensities $u_{R M S}^{\prime}$ and $v_{R M S}^{\prime}$ over the flow domain, versus suction coefficient, at $R e=90$.

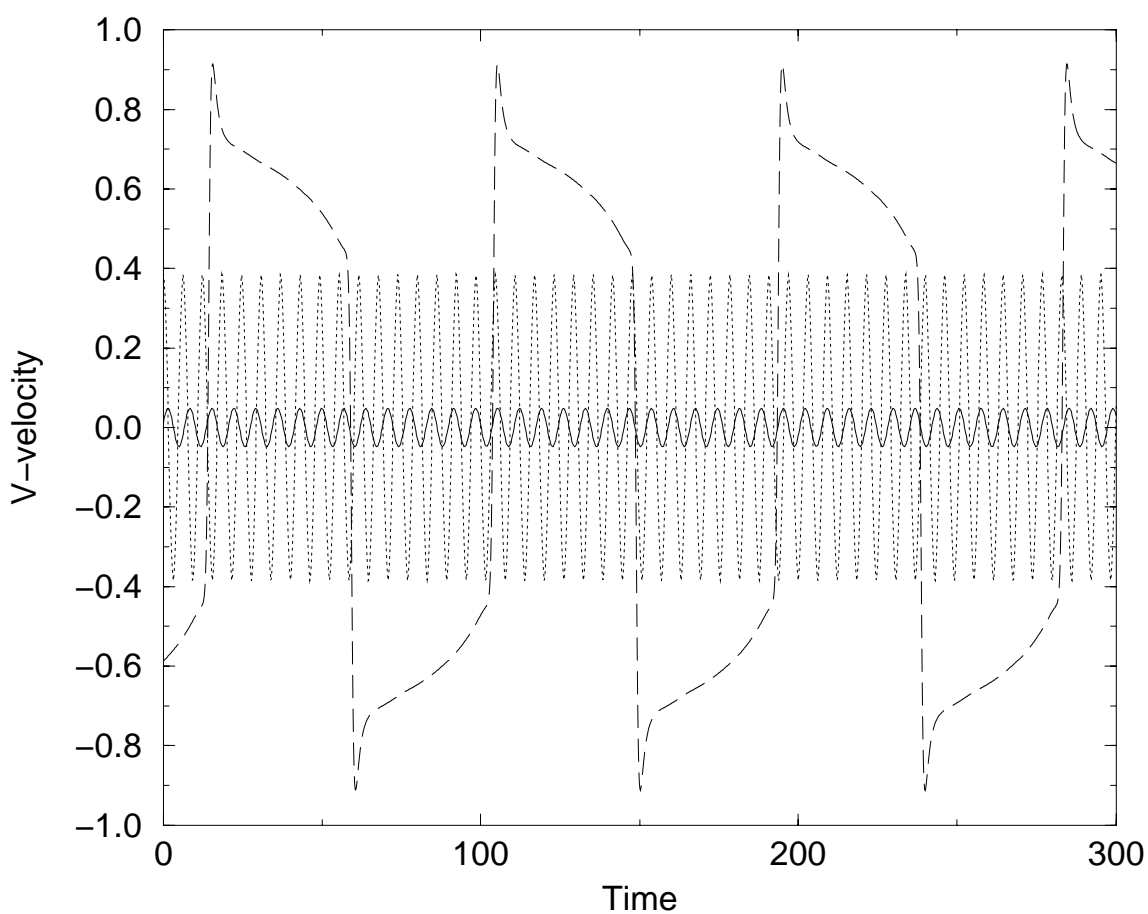

Figure 6: Time history of $\mathrm{V}$-velocity on the limit cycle at point $\mathrm{A}$ for $C_{\text {suc }}=--0.10, \cdots 0.0$, and $---0.85, R e=90$. 


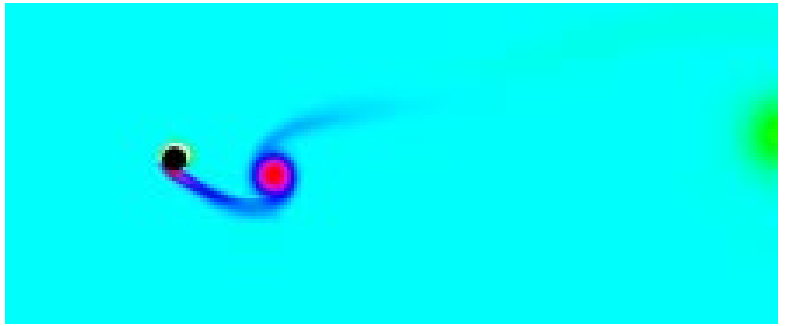

$t=0$

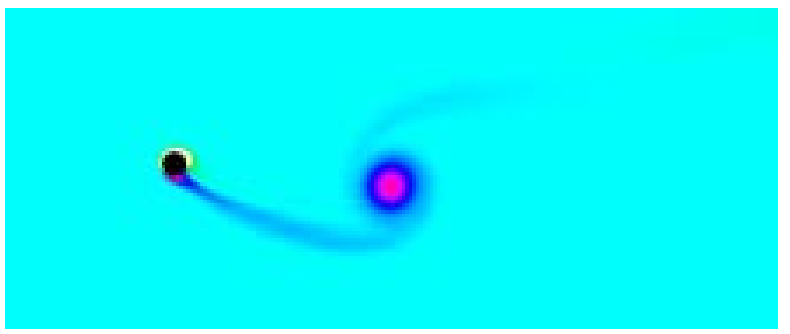

$t=T_{0} / 8$

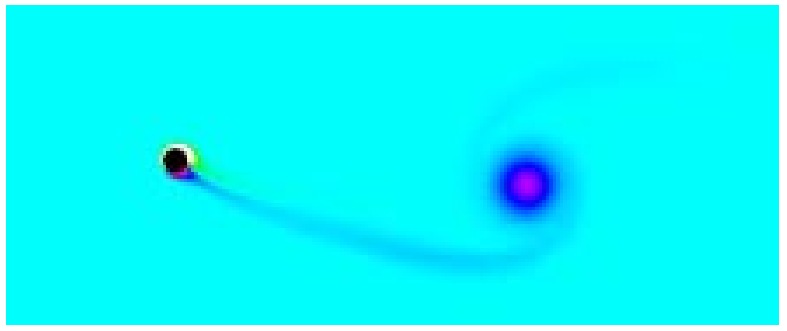

$t=2 \mathrm{~T}_{0} / 8$

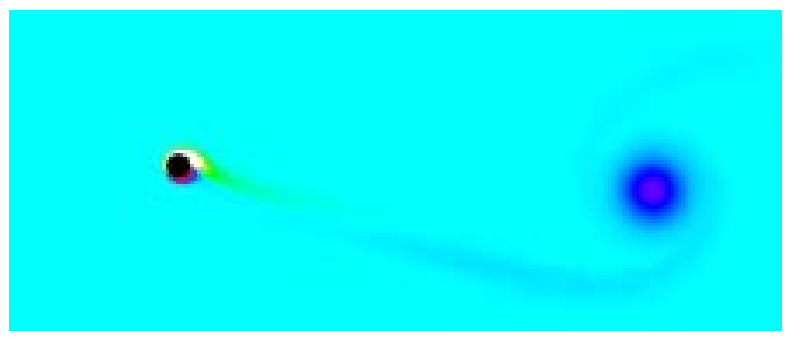

$t=3 \mathrm{~T}_{0} / 8$

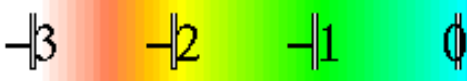

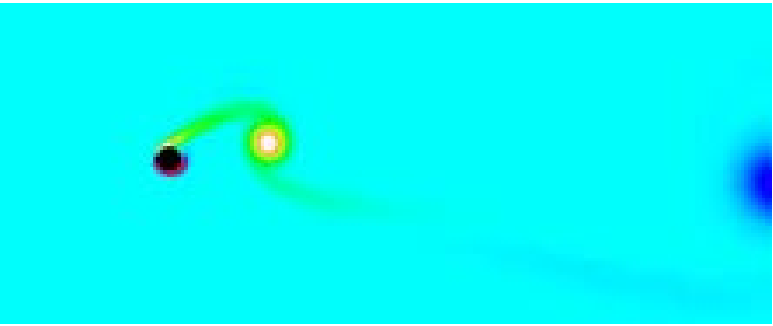

$t=4 T_{0} / 8$

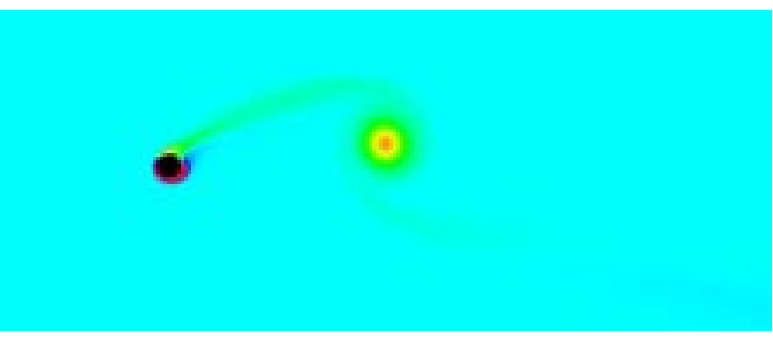

$t=5 \mathrm{~T}_{0} / 8$

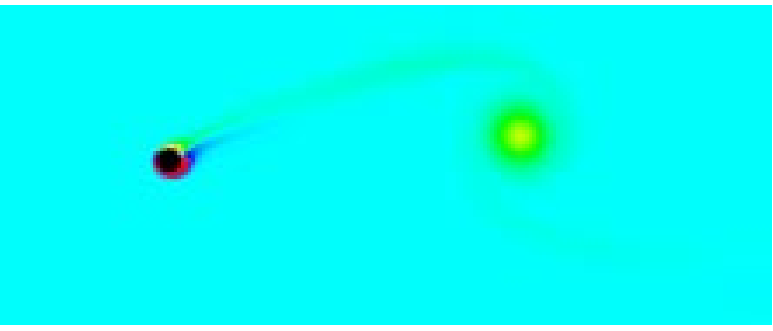

$t=6 T_{0} / 8$

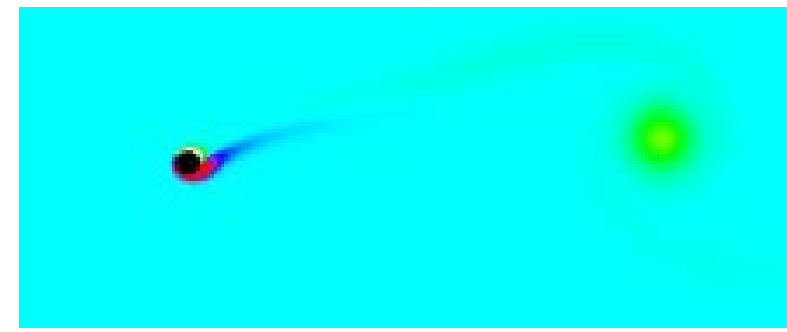

$t=7 \mathrm{~T}_{0} / 8$

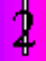

Figure 7: Time evolution of vorticity over one shedding period $\left(T_{0}\right) ; R e=80, C_{\text {suc }}=0.8$. 


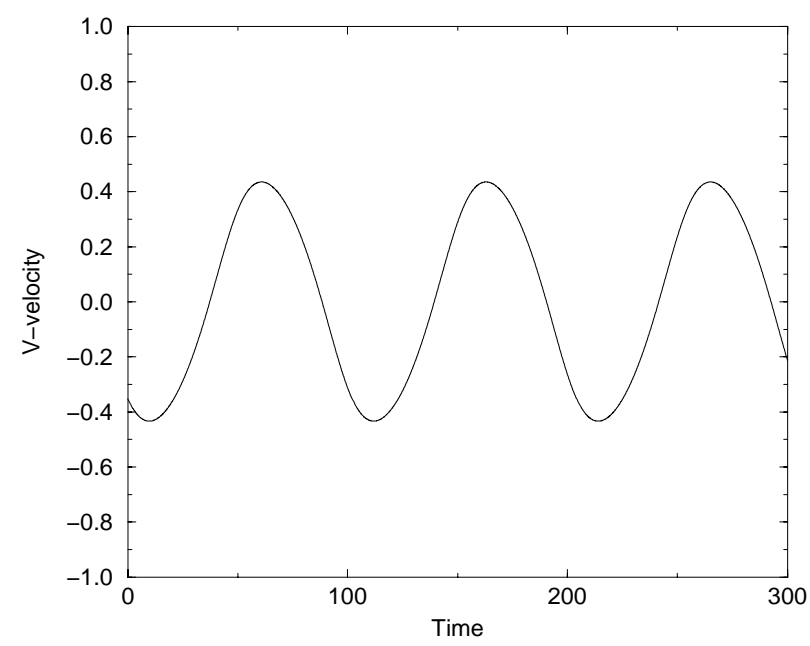

Figure 8: Time history of V-velocity on the limit cycle at point $\mathrm{A}$ for $C_{\text {suc }}=1.58, R e=30$.

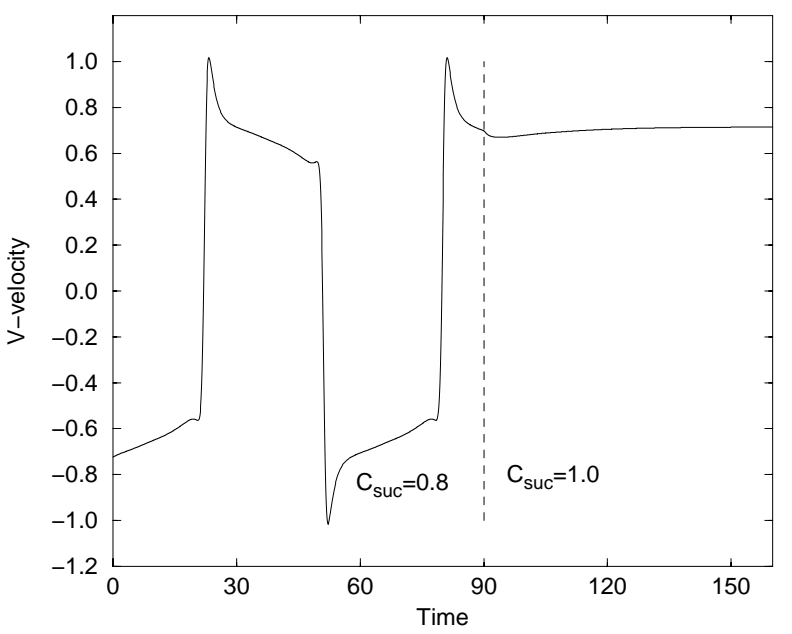

Figure 9: Wake transient (V-velocity at point A) from $C_{\text {suc }}=0.80$ to $C_{\text {suc }}=1.0 ; R e=90$.

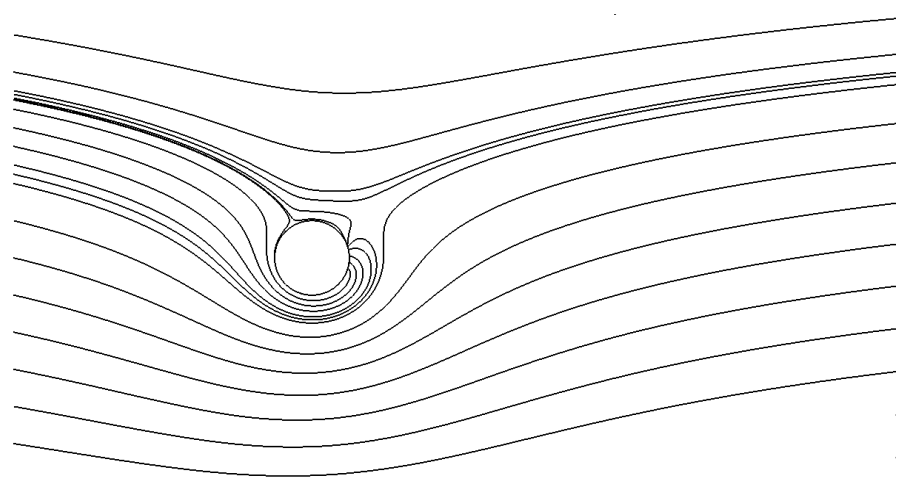

Figure 10: Streamline pattern of the time-asymptotic flow state for $R e=90, C_{\text {suc }}=1.0$. 


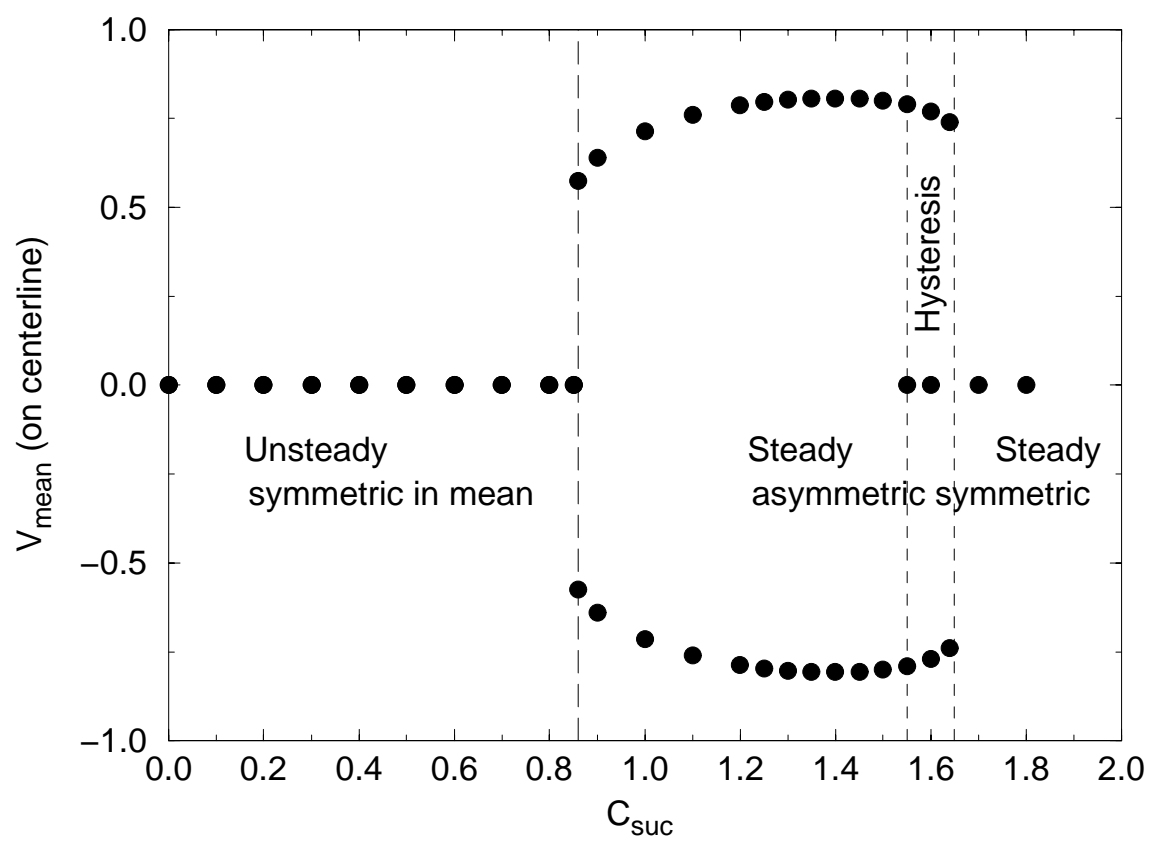

Figure 11: Bifurcation diagram at $R e=90$ : time-averaged V-velocity at centerline point A versus suction coefficient, $C_{\text {suc }}$.

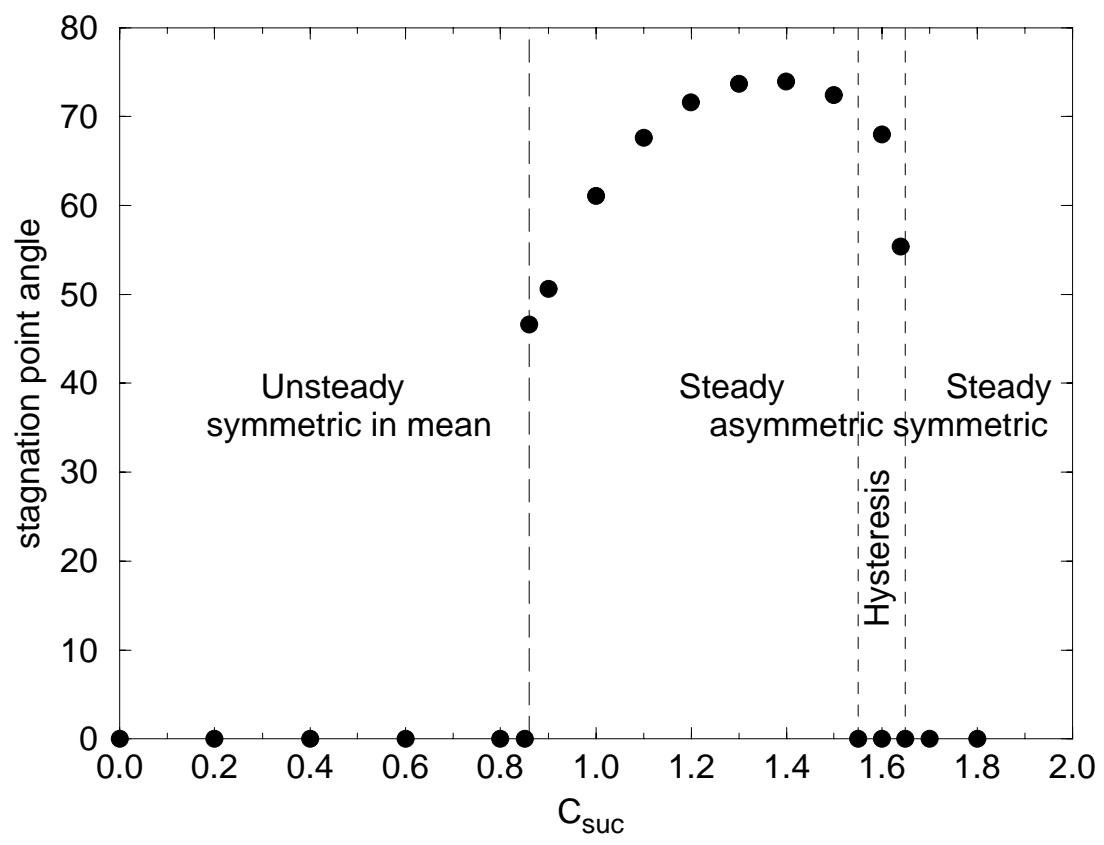

Figure 12: Deflection of front stagnation point (angle in degrees) with respect to the centerline, versus suction coefficient, $C_{\text {suc }}$, for $R e=90$. 


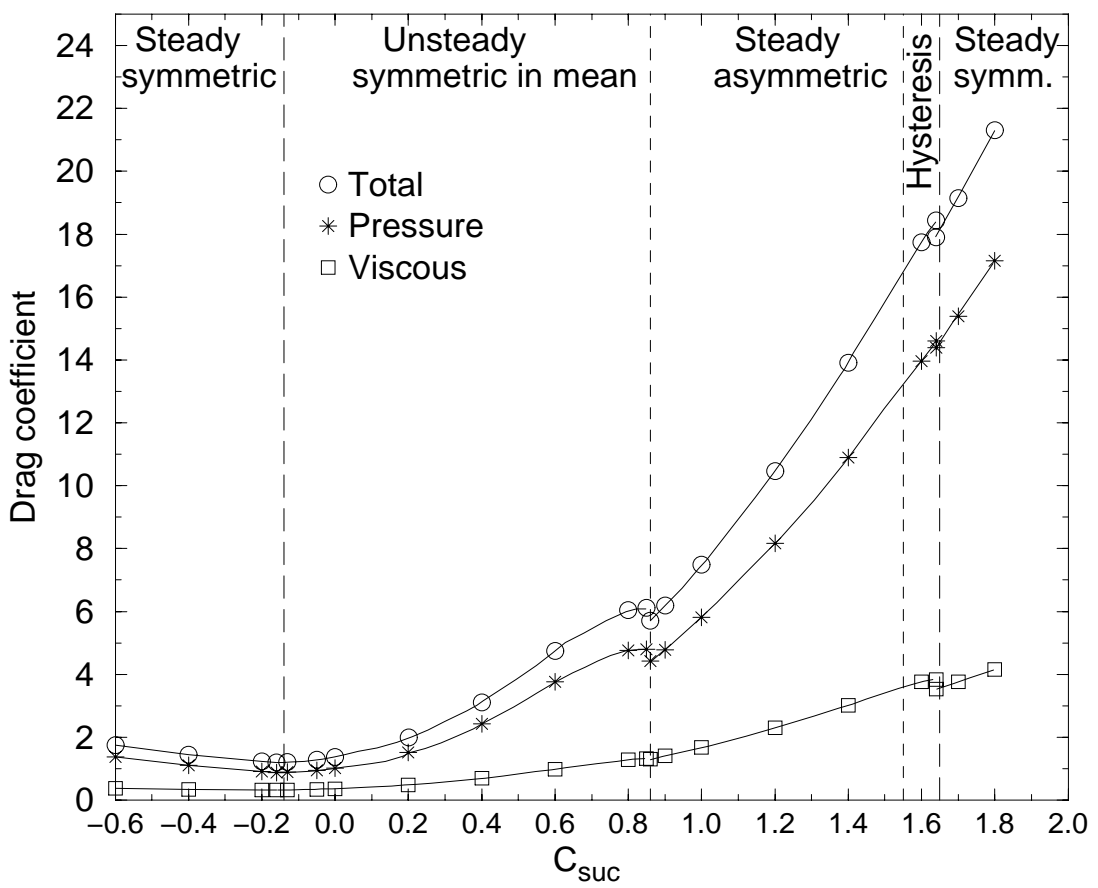

Figure 13: Time-averaged drag coefficient values versus $C_{\text {suc }}$ at $R e=90$

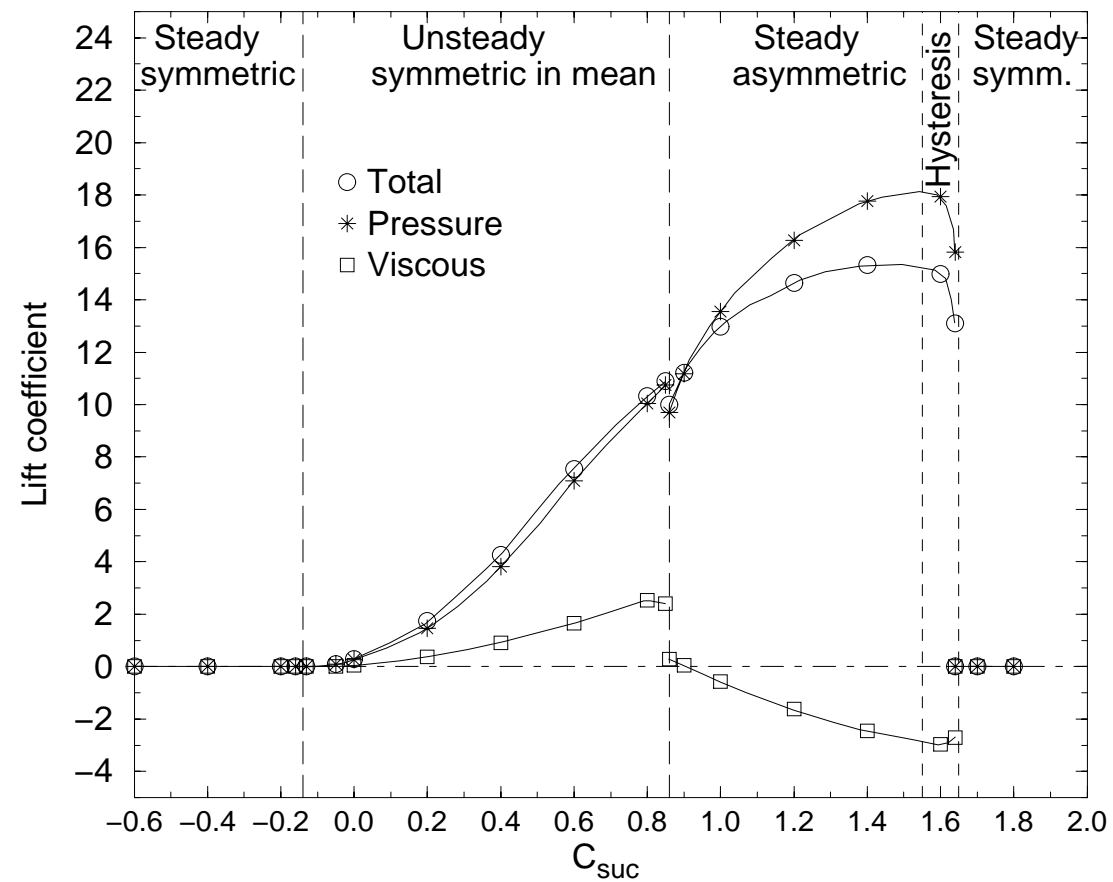

Figure 14: Lift coefficient values versus $C_{s u c}$ at $R e=90$; in the unsteady flow region, the reported values refer to amplitude (half of the difference between maximum and minimum values). 